\title{
LEADER 7: cardiovascular risk profiles of US and European participants in the LEADER diabetes trial differ
}

\author{
Guy E. H. M. Rutten ${ }^{1 *}$, Cees J. Tack ${ }^{2}$, Thomas R. Pieber ${ }^{3}$, Abdurrahman Comlekci ${ }^{4}$, David Dynnes $\varnothing r s t e d^{5}$, \\ Florian M. M. Baeres ${ }^{5}$, Steven P. Marso ${ }^{6}$, John B. Buse ${ }^{7}$ and on behalf of the LEADER Investigators
}

\begin{abstract}
Aims: To determine whether US and European participants in the Liraglutide Effect and Action in Diabetes: Evaluation of cardiovascular outcome Results (LEADER) trial differ regarding risk factors for cardiovascular mortality and morbidity.
\end{abstract}

Methods: Baseline data, stratified for prior cardiovascular disease (CVD), were compared using multivariable logistic regression analysis to establish whether region is an independent determinant of achieved targets for glycated hemoglobin ( $\mathrm{HbA1c})$, blood pressure (BP), and low-density lipoprotein (LDL)-cholesterol.

Results: Independent of CVD history, US participants were more often of non-White origin and had a longer history of type 2 diabetes, higher body weight, and higher baseline $\mathrm{HbA1c}$. They had substantially lower systolic and diastolic $\mathrm{BP}$, and a marginally lower LDL-cholesterol level. Fewer US participants were diagnosed with left ventricular dysfunction. In the largest group of patients, those with prior CVD and the highest cardiovascular risk, US participants were more often female, had a higher waist circumference, and had a decreased estimated glomerular filtration rate, but less frequently prior myocardial infarction or angina pectoris.

Conclusions: There were baseline differences between US and European participants. These differences may result from variation in regional targets for cardiovascular risk factor management, and should be considered in the analysis and reporting of the trial results.

Clinical trial identifier: ClinicalTrials.gov, NCT01179048

Keywords: Type 2 diabetes, External validity, Generalizability, Cardiovascular outcome trial, Heterogeneity

\section{Background}

The Liraglutide Effect and Action in Diabetes: Evaluation of cardiovascular outcome Results (LEADER) trial [1] is one of at least 16 cardiovascular outcome trials in type 2 diabetes (T2D) that will report before 2020 [2]. Driven by regulatory requirements to demonstrate cardiovascular safety, trial sample sizes are large. To recruit 10-20,000 participants per trial, there are 17-27 countries per trial,

\footnotetext{
*Correspondence: g.e.h.m.rutten@umcutrecht.nl

1 Julius Center for Health Sciences and Primary Care, University Medical Center Utrecht, STR 6.131, P.O. Box 85500, 3508, GA, Utrecht, The Netherlands

Full list of author information is available at the end of the article
}

resulting in a wider range of racial and ethnic participation, as well as greater variation in clinical practice patterns than in previous T2D studies [2]. The clinical usefulness of trial data obtained for a particular clinician or policy-maker depends on how well the trial population mirrors the patients in that clinician's practice, or the population for whom the policy is developed. This external validity is determined by inclusion criteria, but also by patients' enrolment, retention, and protocol adherence. The age distribution of trial participants in seven large diabetes trials often did not reflect that of a contemporary British T2D patient population [3]. Most ongoing diabetes cardiovascular outcome trials are being conducted in populations with high cardiovascular 
risk with a long history of T2D [2]. Women and ethnic minorities are under-represented in US-based clinical trials, although these groups have disproportionally higher rates of chronic diseases [4]. The generalizability of results even from well-performed trials may be jeopardized when participants differ from the clinical population with respect to attributes that modify treatment [5]. In a multivariate model, female sex, renal insufficiency, and heart failure were significant predictors of not participating in a trial [6]. Such clinical factors are important because they may modify treatment effects. The table of baseline characteristics in trial reports should focus on baseline factors associated with primary outcomes [7].

Patient enrolment is likely to be dependent on the specific types of investigator practices (generalist, diabetologist, cardiologist, nephrologist, etc.) and on the healthcare systems they operate within. A UK study suggests that selection of study sites should aim to represent the general medical management of T2D patients [8]. It might be questioned whether such a strategy is economically and logistically possible in large global trials.

We seek to examine whether there are important baseline differences in cardiovascular risk markers among LEADER participants enrolled in the US and Europe. This study of these two populations was prompted by the substantial differences between the US and Europe, for example with respect to demographics; cultural norms regarding diet, activity, and other health behaviors; prevailing treatment guidelines for glycemia, blood pressure (BP), lipids, and cardiovascular management; and funding and nature of healthcare systems $[9,10]$. We examined baseline demographic and clinical characteristics of LEADER participants in the US and Europe without a priori hypotheses. Such an exploratory investigation may generate hypotheses about possible heterogeneity [11], and be informative for potential post hoc analyses [11, 12].

\section{Methods}

\section{Study design and participants}

We used baseline data from LEADER (NCT01179048), a phase $3 \mathrm{~b}$, multicenter, international, randomized, double-blind, placebo-controlled clinical trial with longterm follow-up in 9340 patients with T2D. This trial will provide data on the cardiovascular safety of liraglutide compared to placebo. The trial design has been described in more detail previously [1]. Enrolment took place from September 2010 until April 2012. Patients with T2D who were either drug-naive or treated with oral blood glucose-lowering agents or selected insulin regimens (human neutral protamine Hagedorn, long-acting analog, or premixed) were eligible. LEADER enrolled two distinct populations of high-risk patients: (1) patients with prior cardiovascular disease (CVD), $\geq 50$ years old (defined as having one or more of the following cardiovascular comorbidities: prior myocardial infarction, stoke, transient ischemic attack, arterial revascularization, chronic heart failure [New York Heart Association \{NYHA\} class II-III], or chronic renal failure); (2) patients without prior CVD, $\geq 60$ years old with one or more cardiovascular risk factors: micro-albuminuria or proteinuria, hypertension and left ventricular hypertrophy by electrocardiogram (ECG) or imaging, or anklebrachial index $<0.9$. Enrolment of approximately 400 patients with moderate (estimated glomerular filtration rate $\left[\right.$ eGFR] $30-59 \mathrm{~mL} / \mathrm{min} / 1.73 \mathrm{~m}^{2}$ ) and 200 with severe (eGFR $<30 \mathrm{~mL} / \mathrm{min} / 1.73 \mathrm{~m}^{2}$ ) renal impairment was also pre-specified [1].

The present analysis compares baseline data between US and European populations from LEADER, stratified by patients' cardiovascular history. The European population includes all patients enrolled from Austria, Belgium, Czech Republic, Denmark, Finland, France, Germany, Greece, Ireland, Italy, the Netherlands, Norway, Poland, Romania, Serbia, Spain, Sweden, Turkey, and the UK. The trial was designed and conducted according to the declaration of Helsinki and approved by local ethical committees. All subjects signed informed consent before any study procedure was performed.

\section{Outcome measures}

Baseline participant characteristics were recorded at screening visits (Table 1). Concomitant illness was any illness present at the start of the trial, including history of CVD (myocardial infarction, stroke, angina pectoris, arrhythmia, left ventricular dysfunction). Concomitant medication use was defined as use of any medication other than trial product at randomization. Data on blood glucose-lowering medication, the number and type of antihypertensive medication, statin use, other lipid-lowering medication, and aspirin were collected. Height, body weight, and waist circumference were measured without shoes and in light clothing. Systolic BP (SBP) and diastolic BP (DBP) were measured after at least $5 \mathrm{~min}$ rest in the sitting position, with the legs uncrossed and the back and arm supported. Blood samples were collected at randomization and assessed in a central laboratory for glycated hemoglobin (HbA1c), lipids, creatinine, and albumin:creatinine ratio (ACR).

\section{Target levels}

In 2010, 'general' HbA1c goals varied among European countries between $<6.5 \%$ and $<7.0 \%$ ( $<48$ and $<53 \mathrm{mmol} / \mathrm{mol}$ ) [9]. The American Diabetes Association (ADA) recommended in 2010 that less stringent HbA1c goals than the general goal of $7.0 \%(53 \mathrm{mmol} / \mathrm{mol})$ may 
Table 1 Baseline differences between Europe and US patients, stratified by history of prior CVD

\begin{tabular}{|c|c|c|c|c|c|c|}
\hline & \multicolumn{3}{|c|}{ No prior CVD group $(n=1013)$} & \multicolumn{3}{|c|}{ Prior CVD group $(n=4996)$} \\
\hline & Europe $(n=599)$ & USA $(n=414)$ & $P$ value & Europe $(n=2922)$ & USA $(n=2074)$ & $P$ value \\
\hline Gender, female $(n, \%)$ & $223(37.2)$ & $177(42.8)$ & 0.077 & $857(29.3)$ & $710(34.2)$ & 0.001 \\
\hline Age, years (mean, SD) $(n, \%)$ & $66.0(5.0)$ & $66.1(5.6)$ & 0.783 & $64.6(7.5)$ & $64.3(7.7)$ & 0.166 \\
\hline $50-59$ & $10(1.7)$ & $8(1.9)$ & & $798(27.3)$ & $589(28.4)$ & \\
\hline $60-69$ & $454(75.8)$ & $310(74.9)$ & & $1364(46.7)$ & $971(46.8)$ & \\
\hline $70-79$ & $127(21.2)$ & $84(20.3)$ & 0.355 & $694(23.8)$ & $451(21.7)$ & 0.240 \\
\hline $80-89$ & $8(1.3)$ & $12(2.9)$ & & $65(2.2)$ & $62(3.0)$ & \\
\hline $90-99$ & $0(0)$ & $0(0)$ & & $1(0.0)$ & $1(0.0)$ & \\
\hline Diabetes duration, years (mean, SD) & $11.2(6.8)$ & $13.2(7.9)$ & $<0.001$ & $11.7(7.6)$ & $13.5(8.8)$ & $<0.001$ \\
\hline \multicolumn{7}{|l|}{ Race $(n, \%)$} \\
\hline Asian & $3(0.5)$ & $9(2.2)$ & & $25(0.9)$ & $37(1.8)$ & \\
\hline Black & $2(0.3)$ & $101(24.4)$ & $<0.001$ & $14(0.5)$ & $366(17.6)$ & $<0.001$ \\
\hline White & $592(98.8)$ & $295(71.3)$ & & $2871(98.3)$ & $1622(78.2)$ & \\
\hline Other & $2(0.3)$ & $9(2.2)$ & & $12(0.4)$ & $49(2.4)$ & \\
\hline Body weight, kg (mean, SD) & $94.0(18.1)$ & $98.9(23.4)$ & $<0.001$ & $93.6(18.6)$ & $101.1(21.8)$ & $<0.001$ \\
\hline $\mathrm{BMI}, \mathrm{kg} / \mathrm{m}^{2}$ (mean, SD) $(n, \%)$ & $33.0(5.6)$ & $34.4(6.9)$ & $<0.001$ & $32.7(5.8)$ & $34.6(6.8)$ & $<0.001$ \\
\hline $18-25$ & $25(4.2)$ & $24(5.8)$ & & $181(6.2)$ & $96(4.6)$ & \\
\hline $25-30$ & $176(29.4)$ & $91(22.0)$ & & $843(28.9)$ & $438(21.1)$ & \\
\hline $30-35$ & $216(36.1)$ & $125(30.2)$ & $<0.001$ & $1024(35.0)$ & $686(33.1)$ & $<0.001$ \\
\hline $35-40$ & $113(18.9)$ & $91(22.0)$ & & $554(19.0)$ & $478(23.0)$ & \\
\hline$>40$ & $69(11.5)$ & $83(20.0)$ & & $316(10.8)$ & $375(18.1)$ & \\
\hline Waist circumference, cm (mean, SD) & $112.2(15.5)$ & $114.1(18.4)$ & 0.072 & $111.2(15.3)$ & $114.5(17.0)$ & $<0.001$ \\
\hline $\mathrm{HbA1c}$, \% (mean, SD) & $8.4(1.3)$ & $8.9(1.6)$ & $<0.001$ & $8.3(1.3)$ & $8.8(1.5)$ & $<0.001$ \\
\hline \multicolumn{7}{|l|}{$\mathrm{HbA} 1 \mathrm{c}$ at target $(n, \%)$} \\
\hline$\leq 7.5 \%$ & $186(31.1)$ & $68(16.4)$ & $<0.001$ & $880(30.1)$ & $481(23.2)$ & $<0.001$ \\
\hline$\leq 7 \%$ & $49(8.2)$ & $18(4.3)$ & 0.016 & $264(9.0)$ & $157(7.6)$ & 0.066 \\
\hline SBP, mmHg (mean, SD) & $144.1(17.5)$ & $135.5(17.1)$ & $<0.001$ & $140.0(18.4)$ & $133.3(18.5)$ & $<0.001$ \\
\hline DBP, mmHg (mean, SD) & $81.0(9.7)$ & $76.0(9.5)$ & $<0.001$ & $78.5(10.2)$ & $74.7(10.5)$ & $<0.001$ \\
\hline $\begin{array}{l}\text { BP at target ( } n, \%) \text { (Europe < 140/80 and USA 130/80 } \\
\text { mmHg) }\end{array}$ & $146(24.4)$ & $134(32.4)$ & 0.005 & $974(33.3)$ & $800(38.6)$ & $<0.001$ \\
\hline LDL, mmol/L (mean, SD) & $2.5(0.9)$ & $2.3(0.9)$ & $<0.001$ & $2.3(0.9)$ & $2.2(0.9)$ & 0.113 \\
\hline At target $(n, \%)$ & $319(53.3)$ & $273(65.9)$ & $<0.001$ & $1934(66.2)$ & $757(36.5)$ & $<0.001$ \\
\hline $\mathrm{HDL}$, mmol/L (mean, SD) & $1.3(0.3)$ & $1.2(0.4)$ & 0.104 & $1.2(0.3)$ & $1.2(0.3)$ & 0.008 \\
\hline Triglyceride, mmol/L (mean, SD) & $2.0(1.3)$ & $2.0(2.0)$ & 0.749 & $2.1(1.8)$ & $2.0(1.5)$ & 0.038 \\
\hline Creatinine, $\mu \mathrm{mol} / \mathrm{L}$ (mean, SD) & $71.6(14.6)$ & $73.1(15.6)$ & 0.106 & $85.4(31.2)$ & $94.7(38.2)$ & $<0.001$ \\
\hline $\mathrm{ACR}, \mathrm{mg} / \mathrm{mmol}($ mean, SD) & $10.6(30.5)$ & $14.8(50.5)$ & 0.135 & $16.4(57.3)$ & $26.5(82.0)$ & $<0.001$ \\
\hline eGFR (mean, SD) & $90.0(24.0)$ & $90.3(20.5)$ & 0.847 & $81.3(26.8)$ & $74.8(27.1)$ & $<0.001$ \\
\hline \multicolumn{7}{|l|}{ Renal function, eGFR $\left(\mathrm{mL} / \mathrm{min} / 1.73 \mathrm{~m}^{2)}(n, \%)\right.$} \\
\hline Normal $(\geq 90)$ & $268(44.7)$ & $181(43.7)$ & & $1079(36.9)$ & $591(28.5)$ & \\
\hline Mild decline (60-90) & $331(55.3)$ & $233(56.3)$ & 0.748 & $1191(40.8)$ & $809(39.0)$ & $<0.001$ \\
\hline Moderate decline (30-60) & $0(0.0)$ & $0(0.0)$ & & $605(20.7)$ & $625(30.1)$ & \\
\hline Severe decline $(<30)$ & $0(0.0)$ & $0(0.0)$ & & $47(1.6)$ & $49(2.4)$ & \\
\hline Micro-albuminuria ( $n, \%)$ & $246(41.1)$ & $233(56.3)$ & $<0.001$ & $347(11.9)$ & $248(12.0)$ & 0.930 \\
\hline Macro-albuminuria ( $n, \%)$ & $31(5.2)$ & $65(15.7)$ & $<0.001$ & $77(2.6)$ & $66(3.2)$ & 0.253 \\
\hline Myocardial infarction ( $n, \%)$ & $0(0)$ & $0(0)$ & & $567(19.4)$ & $321(15.5)$ & $<0.001$ \\
\hline Stroke $(n, \%)$ & $0(0)$ & $0(0)$ & & $434(14.9)$ & $289(13.9)$ & 0.363 \\
\hline Angina pectoris $(n, \%)$ & $0(0)$ & $5(0.8)$ & 0.062 & $1114(38.1)$ & $617(29.7)$ & $<0.001$ \\
\hline Arrhythmia (n, \%) & $57(9.5)$ & $45(10.9)$ & 0.482 & $479(16.4)$ & $394(19.0)$ & 0.017 \\
\hline Left ventricle dysfunction $(n, \%)$ & $188(31.4)$ & $56(13.5)$ & $<0.001$ & $702(24.0)$ & $379(18.3)$ & $<0.001$ \\
\hline
\end{tabular}


Table 1 continued

\begin{tabular}{|c|c|c|c|c|c|c|}
\hline & \multicolumn{3}{|c|}{ No prior CVD group $(n=1013)$} & \multicolumn{3}{|c|}{ Prior CVD group $(n=4996)$} \\
\hline & Europe $(n=599)$ & USA $(n=414)$ & $P$ value & Europe $(n=2922)$ & USA $(n=2074)$ & $P$ value \\
\hline \multicolumn{7}{|l|}{ Smoking $(n, \%)$} \\
\hline Current & $83(13.9)$ & $57(13.8)$ & & 407 (13.9) & $278(13.4)$ & \\
\hline Never & $248(41.4)$ & $187(45.2)$ & 0.455 & $946(32.4)$ & $754(36.4)$ & 0.013 \\
\hline Previous & $268(44.7)$ & $170(41.1)$ & & $1569(53.7)$ & $1042(50.2)$ & \\
\hline \multicolumn{7}{|l|}{ Pre-trial treatment $(n, \%)$} \\
\hline None/diet & $16(2.7)$ & $41(9.9)$ & & $102(3.5)$ & $226(10.9)$ & \\
\hline Insulin only & $19(3.2)$ & $22(5.3)$ & $<0.001$ & $200(6.8)$ & $210(10.1)$ & $<0.001$ \\
\hline OADs only & $380(63.4)$ & $213(51.4)$ & & $1624(55.6)$ & $973(46.9)$ & \\
\hline Insulin + OADs & $184(30.7)$ & $138(33.3)$ & & $996(34.1)$ & $665(32.1)$ & \\
\hline \multicolumn{7}{|c|}{ Number of oral blood glucose-lowering drugs ( $n, \%)$} \\
\hline No OAD & $219(36.6)$ & $201(48.6)$ & & $1298(44.4)$ & $1101(53.1)$ & \\
\hline $1 \mathrm{OAD}$ & $155(25.9)$ & $83(20.0)$ & 0.424 & $707(24.2)$ & $428(20.6)$ & 0.013 \\
\hline 2 OADs & $199(33.2)$ & $109(26.3)$ & & $826(28.3)$ & $463(22.3)$ & \\
\hline$>2$ OADs & $26(4.3)$ & $21(5.1)$ & & $91(3.1)$ & $82(4.0)$ & \\
\hline BP-lowering medication $(n, \%)$ & $517(86.3)$ & $360(87.0)$ & 0.767 & $2729(93.4)$ & $1930(93.1)$ & 0.639 \\
\hline \multicolumn{7}{|c|}{ Number of antihypertensive medications ( $n, \%)$} \\
\hline 0 & $82(13.7)$ & $54(13.0)$ & & $193(6.6)$ & $144(6.9)$ & \\
\hline 1 & $225(37.6)$ & $182(44.0)$ & 0.018 & $731(25.0)$ & $607(29.3)$ & 0.001 \\
\hline 2 & $172(28.7)$ & $126(30.4)$ & & $1315(45.0)$ & $920(44.4)$ & \\
\hline 3 & $93(15.5)$ & $44(10.6)$ & & $533(18.2)$ & $324(15.6)$ & \\
\hline 4 & $27(4.5)$ & $8(1.9)$ & & $150(5.1)$ & $79(3.8)$ & \\
\hline Statin $(n, \%)$ & $362(60.4)$ & $298(72.0)$ & $<0.001$ & $2369(81.1)$ & $1704(82.2)$ & 0.330 \\
\hline Other lipid-lowering drugs ( $n, \%)$ & $2(0.3)$ & $11(2.7)$ & 0.001 & $39(1.3)$ & $44(2.1)$ & 0.032 \\
\hline Aspirin (n, \%) & $192(32.1)$ & $198(47.8)$ & $<0.001$ & $1934(66.2)$ & $1441(69.5)$ & 0.014 \\
\hline
\end{tabular}

$A C R$ albumin:creatinine ratio, $B M I$ body mass index, $B P$ blood pressure, $C V D$ cardiovascular disease, $D B P$ diastolic blood pressure, $e G F R$ estimated glomerular filtration rate, $H D L$ high-density lipoprotein, $L D L$ low-density lipoprotein, $O A D$ oral antidiabetic drug, $S B P$ systolic blood pressure, $S D$ standard deviation

$P$ values are for difference between region (Europe vs. USA) on covariates ( $t$ test) and factors (Chi square)

be appropriate for patients with advanced macrovascular complications [10]. An ADA/European Association for the Study of Diabetes position statement stated that HbA1c goals between 7.5 and $8.0 \%$ (58 and $64 \mathrm{mmol} /$ $\mathrm{mol})$, or even slightly higher, are appropriate for patients with established vascular complications or extensive comorbid conditions [13]. Previously, SBP and DBP targets also differed among European countries, between $<130$ and $140 \mathrm{mmHg}$ and $<80$ or $<85 \mathrm{mmHg}$, respectively [9]. Until 2013, the ADA recommended a BP target of <130/80 mmHg; in 2014, US guidance on BP in diabetes was relaxed to $<140 /<80 \mathrm{mmHg}$ [14]. Similarly, at the onset of the study in 2010, low-density lipoprotein (LDL)-cholesterol targets differed between European countries, ranging from $<100 \mathrm{mg} / \mathrm{dL}(2.6 \mathrm{mmol} / \mathrm{L})$ and $115 \mathrm{mg} / \mathrm{dL}$ (3 mmol/L) for primary prevention [9]. At that time, the LDL target for US participants was $<100 \mathrm{mg} / \mathrm{dL}(<2.6 \mathrm{mmol} / \mathrm{L}$ ) (no prior CVD) or $<70 \mathrm{mg} /$ $\mathrm{dL}(<1.8 \mathrm{mmol} / \mathrm{L})$ (prior CVD) [10], with the latter goal termed 'an option' in the 2014 recommendations [14].
For the present study, we defined two different HbA1c targets: $\leq 7.0 \%$ and $\leq 7.5 \%$ ( $\leq 53$ and $\leq 58 \mathrm{mmol} / \mathrm{mol}$ ); for both CVD strata and both regions [15]. BP at target was defined as $<140 /<80$ and $<130 /<80 \mathrm{mmHg}$ for European and US participants, respectively. LDL-cholesterol at target was defined as $<100 \mathrm{mg} / \mathrm{dL}(<2.6 \mathrm{mmol} / \mathrm{L})$ for European participants and $<100 \mathrm{mg} / \mathrm{dL}(<2.6 \mathrm{mmol} / \mathrm{L}$ ) (no prior CVD group) or $<70 \mathrm{mg} / \mathrm{dL}(<1.8 \mathrm{mmol} / \mathrm{L})$ (prior CVD group) for US participants.

\section{Statistical analyses}

All analyses were performed using SAS software (version 9.3). Continuous variables were reported as means with standard deviations, categorical variables as numbers and percentages. Because a patient's history of CVD is likely to be related to the cardiovascular outcome of the LEADER trial, the analysis of differences between regions (US vs. Europe) was stratified by prior and no prior CVD. We used t-tests for continuous variables or Chi square test for class variables. Logistic regression analyses were 
then used to determine whether region (Europe/US) was an independent determinant of achieving target for HbA1c, BP, and LDL-cholesterol, respectively. These analyses were adjusted for several well-known possible confounding variables: age, sex, diabetes duration, race, pre-trial diabetes treatment, body mass index (BMI) category, and renal function (creatinine, ACR, and eGFR); these variables were included based on subject matter considerations and availability of data. See Tables 2, 3, 4 for details. $P$ values $\leq 0.01$ were considered statistically significant.

\section{Results}

\section{Baseline differences between Europe and US patients:} whole cohort

Irrespective of CVD history, US and European participants differed on several important CVD risk factors and other diabetes complications (Table 1). US participants were more often of non-White origin, had longer T2D duration, had higher body weight, and were more often in the highest BMI categories compared with European participants. Furthermore, US participants had a $0.5 \%$ higher HbA1c and a different distribution of blood glucose-lowering treatments than European participants. Significantly fewer US participants were at $\mathrm{HbA} 1 \mathrm{c}$ target $\leq 7.5 \%(\leq 58 \mathrm{mmol} / \mathrm{mol})$. However, US participants had substantially lower SBP and DBP, and marginally lower LDL-cholesterol level than European participants. Significantly more European than US participants were diagnosed with left ventricular dysfunction.

\section{Baseline differences between Europe and US patients: group without prior CVD}

In the group without prior CVD, US participants were more frequently at BP target (with different target levels in both regions) and more frequently at LDL target (with comparable targets in both regions, but with significantly more US participants on lipid-modifying medications) than participants from Europe. In addition, more US than European participants without prior CVD had micro- or macro-albuminuria, but the percentages of these patients with decreased kidney function did not differ between regions (Table 1).

Table 2 Determinants of being at $\mathrm{HbA} 1 \mathrm{c}$ target at baseline (HbA1c $\leq 7.5 \%)$, stratified by prior CVD status

\begin{tabular}{|c|c|c|c|c|c|c|}
\hline & \multicolumn{3}{|c|}{ No prior CVD group $(n=1013)$} & \multicolumn{3}{|c|}{ Prior CVD group $(n=4996)$} \\
\hline & Odds ratio & $95 \% \mathrm{Cl}$ & $P$ value & Odds ratio & $95 \% \mathrm{Cl}$ & $P$ value \\
\hline Region (Europe as reference group) & 0.475 & $0.316 ; 0.704$ & $<0.001$ & 0.762 & $0.647 ; 0.896$ & 0.001 \\
\hline Age (per year) & 1.015 & $0.981 ; 1.050$ & 0.393 & 1.032 & $1.020 ; 1.043$ & $<0.001$ \\
\hline Sex (male as reference group) & 0.485 & $0.169 ; 1.271$ & 0.148 & 0.834 & $0.685 ; 1.011$ & 0.065 \\
\hline Diabetes duration (per year) & 0.976 & $0.951 ; 1.000$ & 0.055 & 0.976 & $0.967 ; 0.986$ & $<0.001$ \\
\hline \multicolumn{7}{|l|}{ Race (white as reference group) } \\
\hline Asian & 0.970 & $0.139 ; 4.258$ & & 0.598 & $0.257 ; 1.223$ & \\
\hline Black & 1.691 & $0.672 ; 4.244$ & 0.718 & 1.069 & $0.780 ; 1.452$ & 0.266 \\
\hline Other & 1.358 & $0.195 ; 6.005$ & & 0.604 & $0.258 ; 1.248$ & \\
\hline \multicolumn{7}{|c|}{ Pre-trial diabetes treatment (none, diet as reference group) } \\
\hline Insulin + OADs & 1.080 & $0.475 ; 2.710$ & & 0.800 & $0.586 ; 1.102$ & \\
\hline Insulin only & 0.956 & $0.272 ; 3.215$ & 0.543 & 0.532 & $0.349 ; 0.805$ & $<0.001$ \\
\hline OADs only & 1.365 & $0.629 ; 3.312$ & & 1.248 & $0.930 ; 1.693$ & \\
\hline \multicolumn{7}{|l|}{ BMI (18-25 as reference group) $\left(\mathrm{kg} / \mathrm{m}^{2}\right)$} \\
\hline $25-30$ & 0.628 & $0.286 ; 1.446$ & & 1.220 & $0.871 ; 1.730$ & \\
\hline $30-35$ & 0.788 & $0.369 ; 1.780$ & 0.235 & 1.010 & $0.723 ; 1.427$ & 0.049 \\
\hline $35-40$ & 0.619 & $0.273 ; 1.464$ & & 1.036 & $0.729 ; 1.488$ & \\
\hline$>40$ & 1.082 & $0.476 ; 2.575$ & & 0.824 & $0.561 ; 1.219$ & \\
\hline Creatinine, per $\mu \mathrm{mol} / \mathrm{L}$ & 0.966 & $0.915 ; 1.015$ & 0.179 & 0.998 & $0.993 ; 1.002$ & 0.288 \\
\hline $\mathrm{ACR}$, per mg/mmol & 1.002 & $0.997 ; 1.005$ & 0.421 & 1.000 & $0.999 ; 1.002$ & 0.471 \\
\hline eGFR, per $\mathrm{mL} / \mathrm{min} / 1.73 \mathrm{~m}^{2}$ & 0.967 & $0.931 ; 1.000$ & 0.048 & 0.992 & $0.986 ; 0.998$ & 0.007 \\
\hline
\end{tabular}

Logistic multivariable regression modelling the probability of being at $\mathrm{HbA1c}$ target, adjusted for: age, sex, diabetes duration, race, pre-trial diabetes treatment, BMI category and renal function (creatinine, ACR, eGFR)

$A C R$ albumin:creatinine ratio, $B M I$ body mass index, $C I$ confidence interval, $C V D$ cardiovascular disease, eGFR estimated glomerular filtration rate, $O A D$ oral antidiabetic drug

Overall $P$ values relating to the significance of that factor 
Table 3 Determinants of being at BP target (Europe $<140 / 80 \mathrm{mmHg}$, USA $<130 / 80 \mathrm{mmHg}$ ) at baseline stratified by prior CVD status

\begin{tabular}{|c|c|c|c|c|c|c|}
\hline & \multicolumn{3}{|c|}{ No prior CVD group $(n=1013)$} & \multicolumn{3}{|c|}{ Prior CVD group $(n=4996)$} \\
\hline & Odds ratio & $95 \% \mathrm{Cl}$ & $P$ value & Odds ratio & $95 \% \mathrm{Cl}$ & $P$ value \\
\hline Region (Europe as reference group) & 1.662 & $1.150 ; 2.402$ & 0.007 & 1.314 & $1.136 ; 1.520$ & $<0.001$ \\
\hline Age (per year) & 0.959 & $0.928 ; 0.990$ & 0.009 & 0.992 & $0.982 ; 1.002$ & 0.115 \\
\hline Sex (male as reference group) & 0.823 & $0.341 ; 1.707$ & 0.641 & 1.070 & $0.901 ; 1.270$ & 0.438 \\
\hline Diabetes duration (per year) & 1.020 & $0.998 ; 1.042$ & 0.082 & 1.003 & $0.995 ; 1.012$ & 0.472 \\
\hline \multicolumn{7}{|l|}{ Race (white as reference group) } \\
\hline Asian & 1.758 & $0.457 ; 6.795$ & & 0.880 & $0.483 ; 1.560$ & 0.064 \\
\hline Black & 1.070 & $0.517 ; 2.318$ & 0.604 & 0.724 & $0.551 ; 0.946$ & \\
\hline Other & 0.442 & $0.063 ; 1.987$ & & 0.621 & $0.304 ; 1.189$ & \\
\hline \multicolumn{7}{|l|}{ BMI (18-25, reference group) $\left(\mathrm{kg} / \mathrm{m}^{2}\right)$} \\
\hline $25-30$ & 1.217 & $0.577 ; 2.677$ & & 0.889 & $0.655 ; 1.212$ & \\
\hline $30-35$ & 0.913 & $0.436 ; 1.998$ & 0.009 & 0.793 & $0.587 ; 1.077$ & 0.008 \\
\hline $35-40$ & 0.511 & $0.229 ; 1.177$ & & 0.693 & $0.503 ; 0.957$ & \\
\hline$>40$ & 0.650 & $0.283 ; 1.533$ & & 0.623 & $0.442 ; 0.879$ & \\
\hline \multirow{2}{*}{\multicolumn{7}{|c|}{$\begin{array}{l}\text { Number of antihypertensive medications } \\
0 \text { (reference group) }\end{array}$}} \\
\hline & & & & & & \\
\hline 1 & 1.142 & $0.698 ; 1.897$ & & 0.918 & $0.693 ; 1.221$ & \\
\hline 2 & 0.716 & $0.415 ; 1.244$ & 0.096 & 1.010 & $0.772 ; 1.326$ & $<0.001$ \\
\hline 3 & 0.650 & $0.333 ; 1.256$ & & 0.648 & $0.477 ; 0.881$ & \\
\hline 4 & 1.024 & $0.360 ; 2.670$ & & 0.646 & $0.424 ; 0.975$ & \\
\hline Creatinine, per umol/L & 0.986 & $0.942 ; 1.021$ & 0.488 & 1.004 & $1.000 ; 1.008$ & 0.058 \\
\hline ACR, per mg/mmol & 0.992 & $0.983 ; 0.999$ & 0.013 & 0.994 & $0.992 ; 0.996$ & $<0.001$ \\
\hline eGFR,per $\mathrm{mL} / \mathrm{min} / 1.73 \mathrm{~m}^{2}$ & 0.985 & $0.956 ; 1.007$ & 0.239 & 0.998 & $0.992 ; 1.003$ & 0.334 \\
\hline Micro-albuminuria & 0.895 & $0.628 ; 1.275$ & 0.538 & 0.947 & $0.774 ; 1.155$ & 0.592 \\
\hline Macro-albuminuria & 0.918 & $0.507 ; 1.635$ & 0.775 & 1.562 & $1.060 ; 2.287$ & 0.024 \\
\hline
\end{tabular}

Logistic multivariable regression modelling the probability of being at BP target, adjusted for: age, sex, diabetes duration, race, BMI category, number of antihypertensive medications, renal function (creatinine, ACR, eGFR), and micro- and macro-albuminuria

$A C R$ albumin:creatinine ratio; $B M I$ body mass index; $B P$ blood pressure; $C I$ confidence interval; $C V D$ cardiovascular disease; eGFR estimated glomerular filtration rate

Overall $P$ values relating to the significance of that factor

\section{Baseline differences between Europe and US patients: group with prior CVD}

In the largest group of patients, those with prior CVD and the highest cardiovascular risk, US participants were more often female and had a higher waist circumference than participants from Europe. A higher percentage of US participants were at BP target and a lower percentage of US participants with prior CVD attained LDL target than participants from Europe. The numbers of antihypertensive medications per patient were broadly similar, with slightly more US participants receiving none or one and slightly fewer receiving two, three, or four of these medications, compared with European participants. More US than European participants with prior CVD had decreased eGFR. The percentages of participants with a prior myocardial infarction or angina pectoris also differed significantly between regions (Table 1).

\section{Participants with $\mathrm{HbA1c}$ at target}

Among participants without prior CVD, after multivariable adjustment, the odds ratio (OR) for US participants to have $\mathrm{HbA1c} \leq 7.5 \%(\leq 58 \mathrm{mmol} / \mathrm{mol})$ at baseline was 0.475 (95\% confidence interval [CI] 0.316; 0.704) compared to European participants $(P<0.001)$. In the group of participants with prior CVD, the corresponding OR for US participants was 0.762 (95\% CI $0.647 ; 0.896)$ (Table 2). With a target $\leq 7 \%(\leq 53 \mathrm{mmol} / \mathrm{mol})$, the OR for US participants to be at target at baseline was 0.532 (95\% CI $0.260 ; 1.020$ ) (not significant $[N S]$ ) in the no prior CVD group, and 0.839 (95\% CI $0.646 ; 1.084)$ (NS) in the prior CVD group.

\section{Participants with BP at target}

After adjustment for age, sex, race, diabetes duration, BMI class, number of hypertension medications, renal 
Table 4 Determinants for being at LDL target at baseline (Europe $<100 \mathrm{mg} / \mathrm{dL}$, USA $<100 \mathrm{mg} / \mathrm{dL}$ for no prior CVD and $<70 \mathrm{mg} / \mathrm{dL}$ for prior CVD group)

\begin{tabular}{|c|c|c|c|c|c|c|}
\hline & \multicolumn{3}{|c|}{ No prior CVD group $(n=1013)$} & \multicolumn{3}{|c|}{ Prior CVD group $(n=4996)$} \\
\hline & Odds ratio & $95 \% \mathrm{Cl}$ & $P$ value & Odds ratio & $95 \% \mathrm{Cl}$ & $P$ value \\
\hline Region (Europe as reference group) & 1.616 & $1.116 ; 2.355$ & 0.011 & 0.248 & $0.212 ; 0.290$ & $<0.001$ \\
\hline Age (per year) & 1.038 & $1.006 ; 1.072$ & 0.020 & 1.012 & $1.001 ; 1.023$ & 0.032 \\
\hline Sex (male as reference group) & 0.542 & $0.311 ; 1.009$ & 0.053 & 0.535 & $0.444 ; 0.644$ & $<0.001$ \\
\hline Diabetes duration (per year) & 1.011 & $0.989 ; 1.035$ & 0.330 & 1.004 & $0.995 ; 1.013$ & 0.358 \\
\hline \multicolumn{7}{|l|}{ Race (white as reference group) } \\
\hline Asian & 0.632 & $0.136 ; 3.479$ & & 1.226 & $0.664 ; 2.275$ & \\
\hline Black & 0.710 & $0.371 ; 1.339$ & 0.633 & 0.773 & $0.581 ; 1.023$ & 0.226 \\
\hline Other & 1.53 & $0.313 ; 8.783$ & & 0.759 & $0.389 ; 1.451$ & \\
\hline \multicolumn{7}{|l|}{ BMI $\left(18-25\right.$, reference group) $\left(\mathrm{kg} / \mathrm{m}^{2}\right)$} \\
\hline $25-30$ & 0.571 & $0.239 ; 1.298$ & & 1.029 & $0.735 ; 1.438$ & \\
\hline $30-35$ & 0.836 & $0.353 ; 1.884$ & 0.224 & 1.083 & $0.778 ; 1.503$ & 0.472 \\
\hline $35-40$ & 0.879 & $0.361 ; 2.049$ & & 1.105 & $0.782 ; 1.560$ & \\
\hline$>40$ & 0.935 & $0.375 ; 2.246$ & & 1.287 & $0.891 ; 1.856$ & \\
\hline Statin use (no as reference) & 4.669 & $3.352 ; 6.550$ & $<0.001$ & 4.276 & $3.526 ; 5.202$ & $<0.001$ \\
\hline Aspirin use (no as reference) & 1.055 & $0.750 ; 1.482$ & 0.759 & 1.369 & $1.174 ; 1.597$ & $<0.001$ \\
\hline Creatinine, per umol/L & 1.007 & $0.982 ; 1.038$ & 0.607 & 0.995 & $0.991 ; 1.000$ & 0.034 \\
\hline ACR, per mg/mmol & 0.999 & $0.995 ; 1.003$ & 0.485 & 0.999 & $0.997 ; 1.000$ & 0.017 \\
\hline eGFR per mL/min/1.73 m² & 1.006 & $0.993 ; 1.025$ & 0.360 & 0.994 & $0.988 ; 1.000$ & 0.036 \\
\hline
\end{tabular}

Logistic multivariable regression modelling the probability of being at LDL target, adjusted for: age, sex, diabetes duration, race, BMI category, statin use, aspirin use, and renal function (creatinine, ACR, eGFR)

$A C R$ albumin:creatinine ratio, $B M I$ body mass index, $C I$ confidence interval, $C V D$ cardiovascular disease, eGFR estimated glomerular filtration rate, $L D L$ low-density lipoprotein

Overall $P$ values relating to the significance of that factor. Stratified by prior CVD status

function, and well as micro- and macro-albuminuria, US participants were more often at target BP than participants from Europe, irrespective of their CVD history (no prior CVD OR 1.662; $95 \%$ CI 1.150;2.402; $p=0.007$; prior CVD 1.314; $95 \%$ CI 1.136; 1.520; $P<0.001$ ) (Table 3).

\section{Participants with LDL at target}

US participants with no prior CVD were more often at LDL target at baseline, with a multivariable adjusted OR of 1.616 (95 \% CI 1.116; $2.355 ; P=0.011$ ) compared with European participants. In contrast, in participants with prior CVD, US participants were less likely to be at LDL target than European participants (OR 0.248; 95 \% CI $0.212 ; 0.290 ; P<0.001)$. Not surprisingly, statin use was an independent determinant for achieving LDL target, irrespective of whether it was prescribed as primary or secondary CVD prevention (Table 4).

\section{Discussion}

This study identified relevant differences in baseline cardiovascular risk profiles between European and US participants in the LEADER trial. These differences should be considered in the analysis and reporting of the trial results.
LEADER aims to formally assess the cardiovascular safety of liraglutide, as reflected in the number of cardiovascular events. The trial population consists of people with/without history of CVD. The large number of subjects, as well as the number of study sites, will ensure the results reflect wide clinical practice, and thus the robustness of the primary results. However, whether liraglutide is noninferior to placebo [1] in subgroups will at least partially depend on the baseline risk of the participants. The European and US populations in LEADER differed in many factors related to cardiovascular risk, including race, BMI, HbA1c, SBP and DBP, ventricular dysfunction, and, in the group without prior CVD, albuminuria. In some aspects, US participants may have a lower cardiovascular risk because of their substantially lower BP level and lower prevalence of left ventricular dysfunction, but this could be counteracted by higher cardiovascular risk because of higher BMI and HbA1c. The baseline differences identified should be explored in relation to the overall trial results, once reported. Specifically, testing for heterogeneity of the treatment effect and interactions between subgroups would be needed to evaluate whether these differences impact upon the generalizability of the overall trial findings. Thus, these baseline findings should 
be interpreted with caution, but may inform additional analyses. Calculating an individual's 10 -year CVD risk in people without prior CVD may also help identify which patients benefit most from the treatments under study $[15,16]$.

Regional differences are not unique to LEADER: they probably exist in all global trials, and in cardiovascular outcome trials in diabetes in particular. The analysis in this study provides more detail on potential relevant differences, and hence will support interpretation of the final results. The implications of these data, which show differences related to baseline cardiovascular risk management between US and European participants, may also be relevant in shaping future trial designs with similar populations. Such trial designs will need to account for factors pertaining to cardiovascular risk.

Strengths of this study include the large patient groups involved and investigation of the role of the 'region', independently from patient-related anthropometric and disease-related factors. Region, i.e. Europe or US (a cultural-, socio-economic-, and healthcare-related characteristic), was the most important factor associated with participants achieving HbA1c, BP, and LDL targets. Many of the baseline differences between the US and Europe appear driven by variation in regional targets for cardiovascular risk management. However, the role of 'region' may not only be determined by guidelines and physician prescription patterns, but also the sites that enrolled patients, the way they did so, and patient-physician relationships. A recent systematic review and meta-analysis of randomized controlled trials demonstrated that intensive glycemic control in patients with T2D was associated with increases in all-cause mortality, cardiovascular mortality, and severe hypoglycemia in study populations from North America compared with the rest of the world [17]. Its authors emphasized the need for further investigation into these findings [17]. The choice of participating centers can also influence the generalizability of trial results. For example, among $>55,000$ high-risk patients presenting with non-ST-segment elevation acute coronary syndrome at 443 US hospitals, 1397 (2.5\%) patients were enrolled in a clinical trial. The latter were more often cared for by cardiologists at hospitals capable of catheter-based and surgical revascularization-which might explain the high rates of revascularization among clinical trial patients [6]. Such discrepancies in care and patient characteristics would suggest that the data from this and similar cardiovascular studies cannot be extrapolated to the broad type 2 diabetes population. In the global Trial to Evaluate Cardiovascular Outcomes after Treatment with Sitagliptin (TECOS), regional differences were noted, with lower BP and cholesterol levels in US participants, compared to participants both from western and eastern Europe. Treatment target achievement was also associated with the type of prior vascular disease [18]. Therefore, region-specific data about the centers that enrolled patients (for example, academic sites, rural sites etc.), and region-specific loss-to-follow-up data would enable readers to judge the applicability of the trial results for their practice population.

LEADER was undertaken to assess the cardiovascular safety of liraglutide. A large set of exploratory subgroup analyses have been pre-specified to assess the effect of (among others) sex, BMI, region, cardiovascular risk, chronic heart failure, and severe chronic renal failure on the primary composite endpoint [1]. The present study demonstrates the relevance of these planned subgroup analyses and of evaluating any heterogeneity in outcomes. In this respect, it could also be relevant to present detailed data about sites of enrolment and loss to followup. Finally, patient-level meta-analysis of the CVD trials underway would allow for deeper exploration of these issues, as sufficient sample size of many subgroups would be available [19].

\section{Conclusions}

This study demonstrated important baseline differences between US and European participants in the LEADER trial. These differences may be the result of regional variations in targets for cardiovascular risk factor management. These factors should be considered in the analysis and reporting of the trial results and may also be relevant for potential post hoc investigations.

\section{Abbreviations}

ACR: albumin:creatinine ratio; ADA: American Diabetes Association; BMl: body mass index; BP: blood pressure; Cl: confidence interval; CVD: cardiovascular disease; DBP: diastolic blood pressure; ECG: electrocardiogram; eGFR: estimated glomerular filtration rate; HbA1c: glycated hemoglobin; LDL: low-density lipoprotein; LEADER: Liraglutide Effect and Action in Diabetes: Evaluation of Cardiovascular Outcome Results; NS: not significant; NYHA: New York Heart Association; OR: odds ratio; SBP: systolic blood pressure; T2D: type 2 diabetes; TECOS: Trial to Evaluate Cardiovascular Outcomes after Treatment with Sitagliptin.

\section{Authors' contributions}

GEHMR wrote the manuscript. CJT, AC, DDO, FMMB, SM and JB reviewed and edited the manuscript. DDO extracted the data. SM and JB co-chaired the LEADER Steering Committee and were involved in the design and oversight of the trial. DDO and FMMB are the guarantors of this work and take responsibility for the integrity of the data and the accuracy of the data analysis. All authors read and approved the final manuscript.

\footnotetext{
Author details

1 Julius Center for Health Sciences and Primary Care, University Medical Center Utrecht, STR 6.131, P.O. Box 85500, 3508, GA, Utrecht, The Netherlands. ${ }^{2}$ Department of Internal Medicine, Radboud University Medical Center, Nijmegen, The Netherlands. ${ }^{3}$ Division of Endocrinology and Metabolism, Medical University of Graz, Graz, Austria. ${ }^{4}$ Division of Endocrinology, Dokuz Eylul University Medical School, Inciralti, Izmir, Turkey. ${ }^{5}$ Novo Nordisk, Søborg, Denmark. ${ }^{6}$ Department of Internal Medicine, UT Southwestern, Dallas, TX USA. ${ }^{7}$ Department of Medicine, The University of North Carolina School of Medicine, Chapel Hill, NC, USA.
} 


\section{Acknowledgements}

The authors are grateful to Watermeadow Medical, an Ashfield company (supported by Novo Nordisk), for proofreading and editing assistance. Henrik Wachmann, Department of Biostatistics, Novo Nordisk, Denmark, assisted with the statistical analyses; and Rimke Vos, Ph. D. Julius Center University Medical Center Utrecht, assisted with writing part of the 'Research design and methods' section. The LEADER trial is sponsored by Novo Nordisk, Denmark. The authors did not receive any funding for writing this manuscript.

\section{Competing interests}

GEHMR has served on advisory boards for Novo Nordisk and AstraZeneca, served as a consultant for Novo Nordisk, and received research support from Sanofi Aventis. CJT (or the institute with which he is associated) has received research Grants, served as a consultant for, or given lectures organized by Merck, Janssen, AstraZeneca and Novo Nordisk. TRP has served on advisory panels for AstraZeneca/Bristol-Meyers Squibb, Ltd., Eli Lilly, Inc., Novo Nordisk Inc., Roche Diagnostics, and at speaker's bureau for Novo Nordisk, Inc. and AstraZeneca. AC has served as an advisory panel/board member for Novo Nordisk, Sanofi, Eli Lilly, MSD and AstraZeneca. He has received research support from Novo Nordisk, Astra Zeneca, Novartis, MSD and Boehringer Ingelheim. AC has also served at speaker's bureau for Novo Nordisk, Sanofi, Eli Lilly, AstraZeneca, Novartis and MSD. DDO and FMMB are employees of and hold stock in Novo Nordisk. SPM has served as a consultant for Novo Nordisk, and received research support from Novo Nordisk, Terumo, The Medicines Company, AstraZeneca and Bristol-Myers Squibb. JBB is an investigator and/ or consultant without any direct financial benefit under contracts between his employer and the following companies: Amylin, Andromeda, Astellas, AstraZeneca, Boehringer-Ingelheim, Bristol-Myers Squibb, Dance Pharmaceuticals, Elcylex, Eli Lilly, Gl Dynamics, GlaxoSmithKline, Halozyme, Hoffman-LaRoche, Intarcia Therapeutics, Johnson \& Johnson, Lexicon, LipoScience, MacroGencis, Medtronic MiniMed, Merck, Metabolic Solutions Development Company, Metabolon, Metavention, Novartis, Novo Nordisk, Orexigen, Osiris, Pfizer, Quest, Rhythm, Sanofi, Scion NeuroStim, Takeda, Tolerex, Transtech Pharma, Veritas, and Verva. He has received direct payments and stock options from PhaseBio.

\section{Statement of human and animal rights}

All procedures followed were in accordance with the ethical standards of the responsible committee on human experimentation (institutional and national) and with the Helsinki Declaration of 1975, as revised in 2008 (5).

\section{Statement of informed consent}

Informed consent was obtained from all patients for being included in the study.

Received: 23 March 2016 Accepted: 26 May 2016

Published online: 02 June 2016

\section{References}

1. Marso SP, Poulter NR, Nissen SE, Nauck MA, Zinman B, Daniels GH, Pocock S, Steinberg WM, Bergenstal RM, Mann JF, Ravn LS, Frandsen KB, Moses AC, Buse JB. Design of the liraglutide effect and action in diabetes: evaluation of cardiovascular outcome results (LEADER) trial. Am Heart J. 2013;166:823-30

2. Holman RR, Sourij H, Califf RM. Cardiovascular outcome trials of glucose-lowering drugs or strategies in type 2 diabetes. Lancet. 2014:383:2008-17.

3. Saunders C, Byrne CD, Guthrie B, Lindsay RS, McKnight JA, Philip S, Sattar N, Walker JJ, Wild SH, Scottish Diabetes Research Network Epidemiology Group. External validity of randomized controlled trials of glycaemic control and vascular disease: how representative are participants? Diabet Med. 2013;30:300-8.

4. Rivers D, August EM, Sehovic I, Lee Green B, Quin GP. A systematic review of the factors influencing African American's participation in cancer clinical trials. Contem Clin Trials. 2013:35:13-32.

5. Weiss CO, Boyd CM, Wolff J, Leff B. Prevalence of diabetes treatment effect modifiers: the external validity of trials of older adults. Aging Clin Exp Res. 2012;24:370-6.

6. Kandzari DE, Roe MT, Chen AY, Lytle BL, Pollack CV Jr, Harrington RA, Ohman EM, Gibler WB, Peterson ED. Influence of clinical trial enrollment on the quality of care and outcomes for patients with non-ST-segment elevation acute coronary syndromes. Am Heart J. 2005;149:474-81.

7. Assmann SF, Pocock SJ, Enos LE, Kasten LE. Subgroup analysis and other (mis)uses of baseline data in clinical trials. Lancet. 2000;355:1064-9.

8. Gheorghe A, Roberts TE, Ives JC, Fletcher BR, Calvert M. Centre selection for clinical trials and the generalisability of results; a mixed methods study. PLoS One. 2013;8:e56560.

9. Stone MA, Wilkinson JC, Charpentier G, Clochard N, Grassi G, Lindblad U, Müller UA, Nolan J, Rutten GE, Khunti K, GUIDANCE Study Group. Evaluation and comparison of guidelines for the management of people with type 2 diabetes from eight European countries. Diab Res Clin Pract. 2010;87:252-60.

10. American Diabetes Association. Standards of Medical Care in Diabetes-2010. Diabetes Care. 2010;33:S4-10.

11. Kent DM, Rothwell PM, loannidis JPA, Altman DG, Hayward RA. Assessing and reporting heterogeneity in treatment affects in clinical trials; a proposal. Trials. 2011;11:85.

12. Wagner M, Balk EM, Kent DM, Kasiske BL, Ekberg H. Subgroup analyses in randomized controlled trials: the need for risk stratification in kidney transplantation. Am J Transplant. 2009;9:2217-22.

13. Inzucchi SE, Bergenstal RM, Buse JB, Diamant M, Ferrannini E, Nauck M, Peters AL, Tsapas A, Wender R, Matthews DR, American Diabetes Association (ADA), European Association for the Study of Diabetes (EASD). Management of hyperglycemia in type 2 diabetes: a patient-centered approach. Diabetes Care. 2012;35:1364-79.

14. American Diabetes Association. Clinical Practice Recommendation. Diabetes Care. 2014;37:S1

15. Dorresteijn JA, Visseren FL, Ridker PM, Wassink AM, Paynter NP, Steyerberg EW, van der Graaf Y, Cook NR. Estimating treatment effects for individual patients based on the results of randomised clinical trials. BMJ. 2011;343:d5888.

16. van der Leeuw J, Visseren FL, Woodward M, Zoungas S, Kengne AP, van der GraafY, Glasziou P, Hamet P, MacMahon S, Poulter N, Grobbee DE, Chalmers J. Predicting the effect of blood pressure lowering treatment on major cardiovascular events for individual patients with type 2 diabetes mellitus: results from Action in Diabetes and Vascular Disease: Preterax and Diamicron MR Controlled Evaluation. Hypertension. 2015;65:115-21.

17. Sardar P, Udell JA, Chatterjee S, Bansilal S, Mukherjee D, Farkouh ME. Effect of intensive versus standard blood glucose control in patients with type 2 diabetes in different regions of the world: systematic review and meta-analysis of randomized controlled trials. J Am Heart Assoc. 2015;4:e001577.

18. Bethel MA, Green JB, Milton J, Tajar A, Engel SS, Califf RM. Holman RR; TECOS Executive Committee. Regional, age and sex differences in baseline characteristics of patients enrolled in the Trial Evaluating Cardiovascular Outcomes with Sitagliptin (TECOS). Diabetes Obes Metab. 2015:17:395-402.

19. Hardin DS, Rohwer RD, Curtis BH, Zagar A, Chen L, Boye KS, Jiang HH, Lipkovich IA. Understanding heterogeneity in response to antidiabetes treatment: a post hoc analysis using SIDES, a subgroup identification algorithm. J Diabetes Sci Technol. 2013;7:42. 